\title{
Photometric Properties of Vesta
}

\author{
Jian-Yang $\mathbf{L i}^{1}$, L. Jorda ${ }^{2}$, H. U. Keller ${ }^{3}$, N. Mastrodemos ${ }^{4}$, \\ S. Mottola ${ }^{5}$, A. Nathues ${ }^{6}$, C. Pieters ${ }^{7}$, V. Reddy ${ }^{6}$, C. A. Raymond ${ }^{4}$, \\ T. Roatsch ${ }^{5}$, C. T. Russell ${ }^{8}$, B. J. Buratti ${ }^{4}$, S. E. Schroder ${ }^{6}$, \\ M. V. Sykes ${ }^{1}$, T. Titus ${ }^{9}$, F. Capaccioni ${ }^{10}$, M. T. Capria,${ }^{10}$, \\ L. Le Corre $^{6}$, B. W. Denevi ${ }^{11}$, M. De Sanctis ${ }^{12}$, M. Hoffmann ${ }^{6}$ and M. \\ D. Hicks ${ }^{4}$ \\ ${ }^{1}$ Planetary Science Institute \\ email: jyli@psi.edu \\ ${ }^{2}$ Laboratoire d'Astrophysique de Marseille, ${ }^{3}$ University Braunschweig, IGEP, ${ }^{4}$ Jet Propulsion \\ Laboratory, California Institute of Technology, ${ }^{5}$ DLR, Inst. of Planetary Research, ${ }^{6}$ Institute \\ for Solar System Research, Max-Planck, ${ }^{7}$ Brown University, Planetary Geosciences Group, \\ ${ }^{8}$ UCLA, Institute of Geophysics, ${ }^{9}$ US Geological Survey, Astrogeology Science Center, \\ ${ }^{10}$ INAF, Istituto di Astrofisica Spaziale e Fisica Cosmica, ${ }^{11}$ Johns Hopkins University Applied \\ Physics Laboratory, ${ }^{12}$ INAF, Istituto di Astrofisica Spaziale e Fisica Cosmica
}

\begin{abstract}
The Dawn spacecraft orbited Asteroid (4) Vesta for a year, and returned diskresolved images and spectra covering visible and near-infrared wavelengths at scales as high as $20 \mathrm{~m} /$ pix. The visible geometric albedo of Vesta is $\sim 0.36$. The disk-integrated phase function of Vesta in the visible wavelengths derived from Dawn approach data, previous ground-based observations, and Rosetta OSIRIS observations is consistent with an IAU H-G phase law with $\mathrm{H}=3.2 \mathrm{mag}$ and $\mathrm{G}=0.28$. Hapke's modeling yields a disk-averaged single-scattering albedo of 0.50 , an asymmetry factor of -0.25 , and a roughness parameter of $\sim 20 \mathrm{deg}$ at $700 \mathrm{~nm}$ wavelength. Vesta's surface displays the largest albedo variations observed so far on asteroids, ranging from $\sim 0.10$ to $\sim 0.76$ in geometric albedo in the visible wavelengths. The phase function of Vesta displays obvious systematic variations with respect to wavelength, with steeper slopes within the 1- and 2-micron pyroxene bands, consistent with previous ground-based observations and laboratory measurement of HED meteorites showing deeper bands at higher phase angles. The relatively high albedo of Vesta suggests significant contribution of multiple scattering. The non-linear effect of multiple scattering and the possible systematic variations of phase function with albedo across the surface of Vesta may invalidate the traditional algorithm of applying photometric correction on airless planetary surfaces.
\end{abstract}

